\title{
Article
}

\section{Doxorubicin-induced chronic dilated cardiomyopathy-the apoptosis hypothesis revisited}

Egle, Passante, Henirich, Huber, Kankeu, Cynthia and Klarke, Kylie Available at http://clok.uclan.ac.uk/16696/

Egle, Passante, Henirich, Huber, Kankeu, Cynthia and Klarke, Kylie (2016) Doxorubicin-induced chronic dilated cardiomyopathy - the apoptosis hypothesis revisited. Journal of Molecular Medicine. ISSN 0946-2716

It is advisable to refer to the publisher's version if you intend to cite from the work. http://dx.doi.org/10.1007/s00109-016-1494-0

For more information about UCLan's research in this area go to http://www.uclan.ac.uk/researchgroups/ and search for < name of research Group>.

For information about Research generally at UCLan please go to http://www.uclan.ac.uk/research/

All outputs in CLoK are protected by Intellectual Property Rights law, including Copyright law. Copyright, IPR and Moral Rights for the works on this site are retained by the individual authors and/or other copyright owners. Terms and conditions for use of this material are defined in the policies page.

\section{CLoK}

Central Lancashire online Knowledge www.clok.uclan.ac.uk

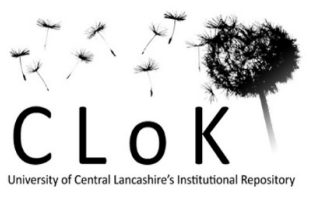




\section{Doxorubicin-Induced Chronic Dilated Cardiomyopathy - The Apoptosis Hypothesis Revisited}

\section{Cynthia Kankeu ${ }^{1}$, Kylie Clarke ${ }^{1}$, Egle Passante ${ }^{2}$ and Heinrich J. Huber ${ }^{1}$}

${ }^{1}$ Department of Cardiovascular Sciences, KU Leuven, 3000 Leuven, Belgium

${ }^{2}$ School of Pharmacy and Biomedical Sciences, Univ. of Central Lancashire, Preston, UK

Running title: Relevance of Apoptosis in Doxorubicin-Induced Dilated Cardiomyopathy

* Corresponding author: Heinrich J. Huber, Ph.D., Department of Cardiovascular Sciences, KU Leuven, Herestraat 49, Box 911, 3000 Leuven, Belgium. Tel + 3216372099 / FAX: + 32476 5021 62, Heinrich.Huber@med.kuleuven.be 


\begin{abstract}
The chemotherapeutic Doxorubicin (DOX) has significantly increased survival rates of pediatric and adult cancer patients. However, $10 \%$ of pediatric cancer survivors will 10-20 years later develop severe Dilated Cardiomyopathy (DCM) and the exact molecular mechanisms of disease progression after this long latency time remain puzzling. We here revisit the hypothesis that elevated apoptosis signaling or its increased likelihood after DOX exposure can lead to an impairment of cardiac function and cause a cardiac dilation. Based on recent literature evidences, we first argue why even little detectable apoptosis may be sufficient to cause a dilated phenotype. We then review findings suggesting that mature cardiomyocytes are protected against DOX-induced apoptosis downstream, but not upstream to Mitochondrial Outer Membrane Permeabilisation (MOMP). This lack of prevention of MOMP-induction then is proposed to alter the metabolic phenotype, induce hypertrophic remodeling and lead to functional cardiac impairment even in absence of cardiomyocyte apoptosis. We further discuss findings that DOX exposure can lead to increased sensitivity to further cardiomyocyte apoptosis, which may cause a gradual loss in cardiomyocytes over time and a compensatory hypertrophic remodeling after treatment, potentially explaining the long lag time in disease onset. We finally note similarities between DOX-exposed cardiomyocytes and apoptosisprimed cancer cells and propose computational systems biology as tool to predict patient individual DOX doses. In conclusion, combining recent findings in rodent hearts and cardiomyocytes exposed to DOX with insights from apoptosis signal transduction allowed us to obtain a molecularly deeper insight in this delayed and still enigmatic pathology of DCM.
\end{abstract}




\section{Key messages}

1. Differentiated cardiomyocyte are protected to post but not pre-MOMP apoptosis

2. MOMP-induction can lead to cardiac hypertrophy and metabolic remodeling after DOX

3. DOX-exposed cardiomyocytes may be more sensitive to apoptosis over life time

4. DOX-exposed cardiomyocytes show similarities to apoptosis-primed cancer cells 


\section{Introduction}

Doxorubicin (DOX) is one of the most widely and successfully applied chemotherapeutic drugs [1-3] and has increased the 5-year survival rates for pediatric cancer patients from $58 \%$ to $80 \%$ within the last 20 years [4]. However, DOX clinical utility and dosage regimens are often limited by severe side effects which can be either acute, sub-acute or late and chronic [5]. While acute and sub-acute toxicity are rare $(1-4 \%)$ and can be managed by adaptation of dosage regimens during, or cessation of, treatment, chronic side effects occur often several years after treatment [6].

The most severe chronic side effect of DOX is chronic dilated cardiomyopathy (DCM). Chronic DCM leads to cardiac dysfunction and eventually congestive heart failure, resulting in the need for heart transplant or even causing death [7]. Chronic DCM occurs in about 10-20\% of cancer survivors often 10-15 years following treatment $[3,8,9]$. As the likelihood of DCM cannot be assessed a priori for an individual patient, the usual dosage of DOX is usually restricted to 60$75 \mathrm{mg} / \mathrm{m}^{2}$ every 3 weeks, hence limiting its efficacy [10].

The late onset of DCM symptoms after cessation of treatment suggests the presence of a slow maladaptive remodeling after initial DOX exposure. Therefore, to understand and mitigate DOX induced chronic DCM, several excellent reviews have focused on the effect of DOXderegulated oxidative metabolism [11, 12], DOX-induced autophagy [13], or on the broader clinical scope of DOX-induced DCM [14]. We here focus on the role of apoptosis to discuss potential molecular underpinnings of a chronic DCM phenotype, paying specific attention on apoptosis alteration after cardiomyocyte differentiation and DOX exposure.

\section{Evidence for cardiomyocyte apoptosis in DOX-induced DCM}

Apoptosis may contribute to DOX-induced DCM and heart failure [12, 15-17] either by acute apoptotic damage of cardiomyocytes or by mechanisms that increase their future apoptosis 
likelihood. However, the relevance of apoptosis in DOX-induced dilated cardiomyopathy is often questioned as the amount of apoptosis in biopsies from patients or from heart transplants following DCM is low compared to necrotic cell death [18]. A suggested reason for the low amount of apoptosis detected in DCM is that apoptosis gradually evolves over time, initiating a slow mechanism of cardiomyocyte hypertrophy over years to replace cardiomyocyte loss [19]. In addition to this, apoptosis is often overshadowed by the presence of secondary necrosis. Secondary necrosis follows the original apoptotic cell death when scavengers of apoptotic debris such as phagocytes operate insufficiently [20] or when ATP levels are too low to guarantee robust apoptosis execution [21], both of which are conditions typical in the impaired and failing heart [22].

Indeed, despite the low detectable amount, several studies suggest a functional role of apoptosis in DCM. In one study, terminal deoxynucleotidyl transferase mediated dUTP-biotin nick end labeling (TUNEL) staining of heart tissue from 21 transplant patients with DCM revealed $0.119 \%$ TUNEL positive cardiomyocytes, indicative of apoptosis. While low, this amount was higher than in patients with ischemic heart disease $(0.075 \%)$ and in control patients $(<0.002 \%)$, and the amount of cardiomyocytes apoptosis positively correlated with disease progression [23]. Moreover, in a transgenic mouse model with constitutively active caspase- 8 only a small detectable elevation of apoptosis only 23 myocytes per $10^{5}$ cells sufficed to induce a dilated cardiomyopathy phenotype [24]. Importantly, this amount is four- to tenfold lower than that measured in three independent studies of failing human hearts. Further, mechanistic support for a role of cardiomyocyte apoptosis in the development of a dilated phenotype following DOX exposure comes for Zhang and colleagues [25]. There, the authors linked the cardiac specific isoform of the canonical target of DOX-induced apoptosis, Topisomerase $2 \beta$ (TOP-2 $\beta$ ) to a DCM phenotype. The authors used a tetracycline-inducible mouse model of TOP-2 $\beta$-deletion specific to the heart. Apoptosis was found to be elevated in TOP- $2 \beta$ wild type mice after DOX- 
treatment, but not in such where TOP-2 $\beta$ was conditionally deleted. Likewise, DOX treatment of TOP-2 $\beta$ wild type mice showed increased left-ventricular end-systolic volume (LVESV), increased diastolic volume (LVEDV), and reduced cardiac ejection fraction (EF). Importantly, these detrimental effects were ablated after DOX exposure in mice with TOP-2 $\beta$-deletion.

\section{Pathways of cardiomyocyte apoptosis relevant to DOX -induced DCM}

We here first describe DOX-induced caspase-dependent apoptosis as found in cancer cells and subsequently discuss evidence of these molecular mechanism that have so far being confirmed in cardiomyocytes ([26], Figure 1). We further note that other proteases [27, 28], metabolic dysfunction or autophagocytic processes [13] may also play a role in regulating cardiomyocyte demise, specifically when canonical caspase activation is blocked or initial injury is too severe $[15]$.

During cancer treatment, DOX firstly intercalates with cellular DNA leading to trapping of topoisomerase II (TOP2), an enzyme that regulates the unfolding of the DNA during replication. Inhibition of TOP2 induces genotoxic stress and the activation of p53 [25, 29]. Transcriptional activity of p53 biases the levels of pro- and anti-apoptotic proteins of the BCL2 family towards the prior by expressing the BCL2 family proteins PUMA and NOXA (Figure 1A), leading to the process of Mitochondrial Outer Membrane Permeabilisation (MOMP; Figure 1B). MOMP leads to the release of further apoptotic proteins, which initiates the so-called downstream apoptosis program, and culminates in caspase activation and cell death (Figure 1C). Besides this genotoxic stress induced, intrinsic pathway of apoptosis, an extrinsic path may also be induced by binding of cell death ligands such as FAS-L to their receptor (Figure 1D). In this step, caspase- 3 is activated either by a positive feedback loop with upstream caspase-8 (socalled type I cells) or after MOMP which is mediated by of the BCL-2 protein BID (type II cells). We further note that MOMP induces an energetic crisis caused by the loss of cytochrome- 
c, which may lead to necrosis or metabolic impairment when caspase-dependent apoptosis is blocked [21, 30, 31].

The above discussed study of Zhang [32] suggested that cardiomyocyte apoptosis after DOX treatment parallels the above mentioned p53-dependent genotoxic stress mechanism from cancer cells by targeting the cardiac specific TOP2 isoform. Indeed, p53-dependency was further suggested by investigating DOX-induced DCM in a mouse model with conditional knock out of the p53-inhibitor MDM4 under presence of p53-null and p53-competent alleles [33]. A potential role of the p53 target PUMA, commonly known to be upregulated after genotoxic stress in cancer cells [34], has also been suggested to be relevant for cardiomyocyte apoptosis in response to DOX as observed for H9C2 cardiomyoblasts [35]. A similar PUMAdependency of cardiomyocyte apoptosis has been found in ischemia-reperfusion model of cultured cardiomyocyte [36]. This pathway would also involve BCL2-protein interaction [37, 38], BAX/BAK-induced MOMP [39] and activation of initiator and activator caspases [24, 39, 40] similar to the above presented canonical intrinsic apoptosis in cancer cells. Likewise, invocation of the extrinsic apoptosis program by DOX via FAS-L or TRAIL ligands has also been detected in cardiomyocytes similar to cancer cells [41, 42], potentially attaining robust caspase-3 activation by invoking MOMP (type II cells, Fig 1).

\section{Are differentiated cardiomyocytes less likely to succumb to DOX-induced apoptosis?}

Due to the above motivated similarity in apoptosis pathways between cancer cells and cardiomyocytes, any strategy that aims to protect cardiomyocytes while killing cancer cells would aim to exploit differences in apoptosis likelihood rather than invoking distinct apoptosis pathways [43-45]. To this end, the Deshmukh lab has shown that differentiated rat 
cardiomyocytes, similar to primary neurons, have down-regulated levels of the pro-apoptotic APAF-1 and increased expression of the anti-apoptotic XIAP compared to less differentiated cells. In agreement with these changes they indeed found a remarkable protection to caspase-3 activation and post-MOMP apoptosis when they added cytochrome $c$ to their cell cultures. Interestingly, this protection was ablated by the XIAP-antagonist SMAC highlighting the balance of pro- and anti-apoptotic proteins in the post-MOMP pathway as decisive for this cardiomyocyte protection $[46,47]$. From their biochemical study, a positive correlation between differentiation status and decreased apoptosis likelihood in response to DOX is hence expected. Evidence for this hypothesis comes from Konorev et al who exposed three cell types with decreasing levels of maturity and differentiation, i.e. adult rat cardiomyocytes (ARCM), neonatal rat cardiomyocytes (NRCM), and rat embryonic H9c2 cardiomyoblasts to DOX [40]. To assess apoptosis, they used dedicated fluorogenic substrates for caspase-3 and caspase- 9 activity. Their results demonstrated that ARCM had markedly lower caspase- 3 and -9 activity than both less differentiated cell types. Likewise, mature NRCMs were more robust to apoptosis than $\mathrm{H} 9 \mathrm{C} 2$ cardiomyoblast. Adding cytochrome $c$ to permeabilised cells confirmed that this protection is mediated by apoptosis downstream to MOMP. In contrast, MOMP induction as evidenced by cytochrome $c$ release revealed a trend that $\mathrm{H} 9 \mathrm{C} 2$ and NRCM cells are more stable to apoptosis than the more differentiated ARCM, suggesting little or the absence of apoptosis protection due to differentiation upstream to MOMP. Decreased apoptosis susceptibility with cardiomyocyte maturity was also proposed in a further study [48]. Comparing post-natal day 7 to day 21 myocardia and exposing them the DOX, these authors confirmed that caspase-3 activity decreased by $>20$ fold and the amount of TUNEL positive cells was significantly reduced, most likely due to a significant reduction of APAF-1 and procaspase-9. However, despite the down-regulation of the upstream, pro-apoptotic protein BAX during maturation, cytochrome $c$ release as indicator for MOMP in response to DOX exposure again did not 
significantly differ between hearts of different maturity. From the above studies we hence conclude that protection against DOX induced apoptosis with increased differentiation status is exercised downstream to MOMP, while, in contrast, processes upstream to MOMP seem to be less protected or show even more susceptibility to MOMP upon differentiation and maturation.

\section{Does DOX exposure change apoptosis likelihood to later somatic cardiomyocyte death?}

Apart from acute cardiomyocyte apoptosis after DOX, a higher cell loss after cessation of treatment is possible when apoptosis likelihood increases after initial DOX exposure. Apoptosis then may then occur gradually over time in response to somatic stimuli resulting in a continuously evolving compensatory cardiomyocyte hypertrophy and extracellular matrix adaptation to replace cell loss [19].

Evidence for increased apoptosis likelihood after DOX exposure comes from studies of FASdependent apoptosis. Specifically, rat-derived embryonic cardiomyocytes, which are normally resistant to FAS-L induced apoptosis [41], may get sensitized to FAS-L when pre-exposed to DOX $[49,50]$. Interestingly, DOX pre-exposure may also induce increased FAS-L released by rat-derived embryonic cardiomyocytes [41], suggesting a DOX-sensitization dependent autocrine/paracrine mechanism for cardiac apoptosis induction. Indeed, elevated FAS-L expression was further found to be a risk factor for idiopathic DCM [51], overload-induced DCM [52] and for inducing apoptosis-mediated cardiac dilation and dysfunction after anti-viral therapy [53], suggesting a role of FAS-L in developing a hypertrophic phenotype.

Besides changes in the secretion of paracrine factors, an increased apoptosis likelihood usually arises by shifting the balance between pro- and anti-apoptotic proteins. In the context of cancer cells this mechanism was coined as apoptosis priming, aiming to explain why cancer cells are more susceptible to chemotherapy than normal cells. Specifically, proponents of the priming 
hypothesis such as the Letai group suggest that cancer cells increase their apoptosis likelihood in response to continuous somatic attack by the immune system $[45,54]$, making them more likely than somatic cells to respond to cytotoxic chemotherapy. Indeed, we observe some parallelism between cancer cells primed by somatic apoptosis [45, 55] and cardiomyocyte being exposed to (sub-lethal) DOX with respect to metabolic programming and apoptosis sensitization (Figure 2).

Evidences that DOX exposure can lead to apoptosis sensitization similar to cancer cell priming comes a study which found a decrease in mRNA expression of the anti-apoptotic BCL-2 and increase of pro-apoptotic BAX [56]. Likewise, in the aforementioned study of Zhang an upregulation of mRNA transcripts was found for the pro-apoptotic gene APAF-1, BAX and FAS$\mathrm{L}$ after DOX exposure in mice with wild type TOP-2 $\beta$. Moreover, re-analyzing their Gene expression Omnibus data set (GSE36239), we also found a transcriptional up-regulation of XAF1, an inhibitor of the anti-apoptotic protein XIAP under the top 200 regulated genes in wild type mice after DOX exposure. How this XAF-1 up-regulation together with de-regulation of other apoptosis genes may lead to elevated apoptosis likelihood in presence of wild type TOP$2 \beta$ mice is illustrated by an in house computational model of apoptosis signal transduction as given and described in Figure 3. Together, these findings suggest an apoptosis priming by DOX on a transcriptional level, making cardiomyocytes more likely to respond to further apoptosis stimuli [25].

Similar as in the case of cardiomyocyte differentiation, however, the change in apoptosis sensitivity after DOX pre-exposure seems to be different between pathways upstream and downstream to MOMP. Hence, increased protection against MOMP after DOX exposure, and hence a counter-priming, is indicated by elevation of the anti-apoptotic protein BCL2 [23], by an increase of BCL2/BAX protein ratio [57] and elevation of the anti-apoptotic gene ARC in rat hearts $[58,59]$. As most of these studies were performed in whole hearts, further studies will 
reveal to what extent DOX primes or anti-primes cardiomyocytes to further pre- or post-MOMP apoptosis or to apoptosis susceptibility to cell death ligands.

\section{Does DOX-induced MOMP render functionally impaired cardiomyocytes even in the absence of apoptosis?}

The above studies suggest that cardiomyocytes acquire robustness against caspase-activation upon differentiation. In contrast, the likelihood of MOMP induction following apoptosis stimuli seems to be unaffected by differentiation, or even higher than in undifferentiated cells $[40,46$, 48]. Moreover, as also outline above, MOMP may be induced by elevated FAS signaling after DOX. We therefore argue that protection against downstream apoptosis in differentiated cardiomyocytes in the presence of MOMP following acute DOX exposure may not be sufficient to guarantee viable, functional cells [60].

The first argument is that cytochrome- $c$ release following MOMP deprives mitochondria of this important electron carrier in the mitochondrial respiration chain. This deprivation induces a bioenergetics crisis [57] consisting of lack of ATP production and failure of ionic homeostasis [61] and can directly lead to necrotic cell death in some cardiomyocytes and hence further cardiac injury. To avoid such necrosis, some cells have developed means to compensate the lack of functional mitochondria by a switch towards increased glycolysis, thereby maintaining ATP levels and ionic homeostasis after MOMP [62] [30]. Evidence for the presence of such a switch also in DOX-exposed cardiomyocytes comes from the observation of a shift from mitochondrial to glycolytic metabolism in the hearts of patients with DOX-induced or idiopathic DCM $[3,5,63,64]$. The presence of such a glycolytic switch is further backed up by the fact that glucose transporter type I (GLUT1, the embryonic isoform[65]) was elevated in rat hearts after DOX and led to higher glucose import [66] to address the higher glucose demand by glycolysis. Together, these findings raise the possibility that DOX-induced MOMP can 
either kill cardiomyocytes by necrosis or invoke glycolytic remodeling. While this remodeling may keep cardiomyocytes viable, they may remain less functional as glycolysis may not sufficiently address the high energy demand in the heart [5, 63, 64] [22].

Another argument that suppression of downstream apoptosis after MOMP is not sufficient to guarantee functional cardiomyocytes is that despite suppression of caspase- 3 activation in differentiated cardiomyocytes, its activity may not be completely abolished after DOX exposure. Indeed, mild, non-lethal caspase-3 activity can lead to structural remodeling such as seen in other large cells [67]. As nicely shown by single cell imaging and quantitative systems biology studies of apoptosis, such sub-lethal caspase-3 activity can arise when tipping the balance for expression of proteins relevant in the post-MOMP pathway from pro-apoptotic to anti-apoptotic ones $[31,68,69]$. Indeed, a recent study also demonstrated that apoptosome dependent, sub-lethal caspase-activity can also lead, and even is essential, in a model of cardiomyocyte hypertrophic remodeling [70]. Specifically using hypertrophy inducing agonists, these study authors demonstrated that caspase- 9 and caspase- 3 were activated in the cardiomyocytes at an extent that was too low to induce apoptosis. Cardiomyocyte hypertrophy was ablated after either blocking caspase- 9 and caspase-3. Likewise, in the rat myocardium the hypertrophic phenotype was attenuated after hypertrophy inducing agonist when caspase- 3 was blocked. Most interestingly, activating caspase-3 with targeted pharmacologic activators (PAC1 compounds) was sufficient to induce cardiomyocyte hypertrophy in the absence of other hypertrophy-inducing stimuli. These findings are consistent with previous findings of a relation between incomplete apoptosis, sub-lethal caspase-3 activity and non-functional cardiomyocytes ('zombie cells') that were found in patients with a DCM phenotype [71]. We therefore propose that structural remodeling of cardiomyocytes after DOX may partly be a consequence of sublethal caspase activation following acute DOX treatment, as differentiated cardiomyocytes show attenuated, but potentially not completely blocked caspase-3 activation after MOMP. 


\section{The apoptosis hypothesis relevant to other pathways in DOX-induced injury}

Whether or not apoptosis is the dominant regulator of DOX-induced DCM remains controversial. Nevertheless, apoptosis has also been implicated in most of the concurring models of DOX-induced DCM. To this end, increased production of ROS is considered to be a major inducer of cardiac malignancy following DOX [12]. ROS is usually thought to be elevated in cardiomyocyte by DOX binding to cardiolipin at the inner mitochondrial membrane, impairing mitochondrial respiration [72]. ROS after DOX exposure can also be induced by altering endothelial nitric oxidase signaling leading further to reduced production of the cardiac vasodilator NO [73]. Both, increased ROS and lack NO can hence directly impair the myocardium and heart muscle compliance even in the absence of apoptosis [74]. Besides this direct impairment of cardiac function, ROS-induced oxidative stress can also induce cardiomyocyte apoptosis [75]. Thereby, oxidative stress has been shown to transcriptionally alter BCL-2 family protein expression, leading to MOMP and cytochrome-c release [12, 76]. Hence, any DOX-dependent apoptosis sensitization as proposed here may exacerbate ROSinduced impairment of cardiac viability. Conversely, it is possible that apoptosis sensitization after DOX exposure results from oxidative stress-induced transcription of pro-apoptotic proteins [77].

The interplay between protective autophagocytic processes and apoptosis was also suggested to be a regulator of DOX-inducted injury $[13,78]$, potentially allowing the alleviation of metabolic stress after MOMP or after other ROS-induced impairment of mitochondria [72]. Indeed, upregulation of the major autophagy inducer Beclin-1 has been observed in cardiomyocytes in a time and dose dependent manner after DOX exposure [78]. Importantly, as Beclin-1 is a substrate of caspase-3, the down-regulation of caspase-3 activity in differentiated cardiomyocytes may guarantee execution of autophagy, therefore alleviating 
cellular metabolic injury. Nevertheless, activity of autophagocytation of mitochondria (mitophagy), which may become damaged after DOX-induced MOMP, has been also shown to be detrimental. To this end, first and recent literature indications point to positive correlation between mitophagy and sensitization to DOX-injury, although the causative role of mitophagy remains yet open $[79,80]$.

\section{Limitations of current studies on the effect of DOX exposure}

Our study is dedicated to the relevance of apoptosis signaling in DOX induced DCM with a focus of how cardiomyocyte sensitivity to apoptosis depends on maturation and differentiation state and to previous exposure to DOX. We have therefore limited our report to the analysis of whole heart tissue and dedicated cardiomyocyte studies. Yet, DOX susceptibility to apoptosis in other relevant cells such as in bovine aortic endothelial cells were also reported and apoptosis may act in a p53-dependent and independent way [81]. Consistent with our focus on cardiomyocytes, we have further limited the discussion of sub lethal caspase-activation to structural remodeling and not considered other potential effects such as caspase-induced cellular hyper-proliferation, potentially relevant in other cardiac cells [82].

\section{Conclusion and Outlook}

In this review we discussed DOX-induced DCM from the perspective of how cardiomyocyte sensitivity to apoptosis depends on differentiation state and previous exposure to DOX. Still, the relation between differentiation, DOX-exposure and apoptosis likelihood requires further investigation of a cardiomyocyte level. This may be done by differentiating H9C2 cardiomyoblast [83], cardiac progenitor cells or inducible pluripotent stem cells and by preexposing the differentiated cells to mild DOX that would not kill the cells, but rather alter their composition of cell death proteins. 
We further note that changes of protein expressions relevant to apoptosis can be subtle and that regulation of proteins in response to differentiation and DOX pre-exposure have to be studied in their interplay. As such example, the studies of Potts [46] and Konorev [40] agreed that postMOMP apoptosis is markedly reduced upon cell maturation, but attributed this effect to different protein regulation patterns. We therefore propose computational signal transduction models to investigate the effect of how combined changes in protein expression levels translate to cardiomyocyte vulnerability. Figure 3 illustrates how such models can be exploited to quantitatively assess apoptosis likelihood in presence and absence of DOX pre-exposure from expression data relevant to apoptosis proteins. Our model illustrates that, while regulation patterns of genes and proteins may be more diffuse between subjects and conditions, their combined action in a defined pathway can lead to a clearer regulation pattern of apoptosis. Indeed, with such quantitative signal transduction models, we were previously able to predict patient specific susceptibility to chemotherapy $[31,44,54,84,85]$. It is therefore expected that these models in combination with biomolecular studies that investigate molecular mediators of apoptosis can further help to identify patient more vulnerable to DOX-induced cardiac apoptosis and DCM. These computer models may further predict targets for secondary treatments such as dexrazoan [86] that reduce cardiomyocyte apoptosis after DOX, while leavings DOX's action in cancer cells intact.

\section{Acknowledgement}

This work has been supported by the Bijzonder Onderzoeksfonds of the KU Leuven (PF/10/014) to H. J. H. 


\section{Figure 1: Canonical apoptosis pathways following DOX exposure}

The canonical pathway of apoptosis following DOX and other topoisomerase inhibitors is depicted. Apoptotic players no yet demonstrated in cardiomyocytes are indicated with a question mark. (A-C) The intrinsic pathway of apoptosis following genotoxic stress by TOP II inhibition is given. (A) Trapping of TOP II after DOX exposure leads to p53-dependent upregulation of the pro-apoptotic BCL-2 protein family member PUMA and NOXA (not yet shown in cardiomyocytes). This upregulation changes the balance of pro- and anti-apoptotic BCL-2 protein family members towards the prior, and culminates in the activation of BAK and BAX to form pores in the mitochondrial outer membrane, leading to MOMP. (B) During MOMP, the pro-apoptotic proteins cytochrome-c and SMAC (?) are released. (C) Downstream apoptosis after MOMP is mediated by the APAF-1 (?) apoptosome-dependent activation of caspase-9 and -3 leading to cell death. Downstream apoptosis is negatively and positively regulated by XIAP and SMAC, respectively. The likelihood of downstream apoptosis was suggested to be reduced after differentiation $[40,46]$ and increased after cardiomyocytes have been exposed by DOX before (cite: Zhang) (D) Extrinsic apoptosis is mediated by cell death ligands such as FAS-L binding to their receptor. This binding leads to a conformational change of the cytosolic moiety of the receptor, recruiting the adaptor protein FADD and caspase-8. Caspase- 8 gets auto-activated by proximity and this process is regulated by long and short isoforms of FLIP. In type I cells, robust caspase-3 activation is initiated its activation and due to a positive feedback with caspase- 8 . In type II cells, MOPM is further initiated by caspase- 8 mediated cleavage and activation of the BCL-2 family protein BID. Cardiomyocytes have been shown to release FAS-L after DOX [41] while getting also sensitized to DOX and respond to it in an auto/paracrine manner [49]. (E) Due to the absence of cytochrome-c in the mitochondrial inter membrane space, MOMP can lead to a bioenergetics crisis with loss of mitochondrial polarization, reduced ATP production, failure of ionic homeostasis. This mechanism may lead 
to necrosis or a maladaptive metabolic phenotype rendering cardiomyocytes less functional [30, 62].

Figure 2: DOX-exposure of cardiomyocyte as analogy to apoptosis priming in cancer cells

Analogies between cardiomyocytes exposed to DOX and cancer cells primed to apoptosis in the body. This hypothesis is linking toxic pre-exposure to apoptosis sensitization, metabolic remodeling and differentiation status. (Left panel) Cardiomyocytes exposed to DOX have a tendency to change from oxidative to glycolytic mechanism and to show a functional impairment $[5,63]$, potentially also associated with a switch to fetal gene expression program [87] \{Hrelia, 2002 \#97\}. Likewise, while cardiomyocytes have acquired apoptosis resistance during differentiation [46], DOX exposure may change the balance of pro- and anti-apoptotic proteins in favor of the latter [25, 88] or sensitize cardiomyocytes to cell death ligands [49]. (Right panel) As an analogy, cancer cells are de-differentiated cells arising from differentiated healthy tissue [43]. On a metabolic level, this de-differentiation is characterized by increased glycolysis and down-regulation of mitochondrial ATP production, potentially by a shift from the mature to the fetal pyruvate-dependent kinase isoform [89] [90]. In addition, as cancer cells are continuously attacked by the immune system, the constant somatic stress makes them more sensitized to undergo chemotherapy-induced apoptosis. This sensitization was coined as apoptosis priming $[45,55]$ and was recently explained by us through systematic change in balance in favor of pro-apoptotic proteins using a systems analysis [54]. 


\section{Figure 3: Computational signal transduction models to translate subtle changes is}

\section{protein and gene expression into apoptosis likelihood}

Illustration of a how a computational signal transduction analysis can quantitatively assess the likelihood of apoptosis downstream to MOMP in mouse hearts in the presence and absence of DOX pre-exposure. This analysis is based on our previously published computer model of postMOMP apoptosis, APOPTO-CELL. As adaptation, we also included the pro-apoptotic protein $\mathrm{XAF}-1$ which we modelled to act in the same way as SMAC, yet to be present initially in the cytosol instead of being released from the mitochondria [31, 68]. As input we used gene expression data from Zhang [25] (GEO data set GSE36239). These authors performed an expression microarray analysis of heart tissue from wild type or TOP 2B -/- mice (inhibiting the canonical pathway of DOX) which they either subjected to PBS (control) or DOX. Hearts from three mice per condition were analyzed. (A) Analysis of expression profiles of apoptosis proteins required as input for APOPTO-CELL. Microarray read outs from GSE36239 were normalized to the average from the three PBS-exposed wild type mice. Results revealed that mice with functional TOP2B and subjected to DOX had either significantly or nearly significantly $(\mathrm{n}=3, * \mathrm{p}<0.05$, two sided t-test) increased XAF-1 levels compared to those mice where DOX was either not applied or its pathway was assumed to be non-functional (Top2b /-). A similar expression trend, albeit showing no significance, was observed for the proapoptotic protein APAF-1. This trend was also present at much smaller extent in the proapoptotic protein caspase-3. Other proteins relevant to APOPTO-CELL did not show an observable trend with expression differences between groups lower than a factor 1.3 and were hence not shown. (B) Analysis pipeline to study change in apoptosis likelihood after wild type and TOP2b -/- mice have been exposed to PBS or DOX. As absolute protein expressions are needed as model input, we assumed a set point for the relevant proteins (caspase-3: $100 \mathrm{nM}$, caspase-9: $10 \mathrm{nM}$, XIAP: $500 \mathrm{mN}$, APAF-1:100 nM, SMAC: $100 \mathrm{nM}, \mathrm{XAF}-1: 100 \mathrm{nM})$. This 
set point was considered to describe the average expressions in the PBS exposed group of three wild type mice. From this, we used the changes in expression levels for each group (see panel A) and for each mouse in the group to estimate sample-individual profiles for above proteins. Using this as model input, APOPTO-CELL calculated the likelihood of cell death after 300 minutes which is an estimate how PBS and DOX pre-exposed mice would react to further apoptosis stimuli. As result, mice with functional TOP $2 \mathrm{~B}$ and exposed to DOX were statistically significantly primed to apoptosis compared to such which were either not exposed to DOX or where its canonical pathway was not functional (TOP2B-/-). 


\section{References}

1. Blum, R.H. and S.K. Carter, Adriamycin. A new anticancer drug with significant clinical activity. Ann Intern Med, 1974. 80(2): p. 249-59.

2. Lefrak, E.A., et al., A clinicopathologic analysis of adriamycin cardiotoxicity. Cancer, 1973. 32(2): p. 302-14.

3. Singal, P.K. and N. Iliskovic, Doxorubicin-induced cardiomyopathy. N Engl J Med, 1998. 339(13): p. 900-5.

4. Ries, L., D. Melbert, and M. Krapcho, SEER Cancer Statistics Review, 1975-2004, N.C. Institute, Editor. 2007: Bethesda.

5. Carvalho, R.A., et al., Metabolic remodeling associated with subchronic doxorubicin cardiomyopathy. Toxicology, 2010. 270(2-3): p. 92-8.

6. Slordal, L. and O. Spigset, Heart failure induced by non-cardiac drugs. Drug Saf, 2006. 29(7): p. 567-86.

7. Lenneman, A.J., et al., Heart transplant survival outcomes for adriamycin-dilated cardiomyopathy. Am J Cardiol, 2013. 111(4): p. 609-12.

8. Lipshultz, S.E., et al., Late cardiac effects of doxorubicin therapy for acute lymphoblastic leukemia in childhood. N Engl J Med, 1991. 324(12): p. 808-15.

9. Lipshultz, S.E., et al., Anthracycline-related cardiotoxicity in childhood cancer survivors. Curr Opin Cardiol, 2014. 29(1): p. 103-12.

10. Von Hoff, D.D., et al., Risk factors for doxorubicin-induced congestive heart failure. Ann Intern Med, 1979. 91(5): p. 710-7.

11. Meredith, A.M. and C.R. Dass, Increasing role of the cancer chemotherapeutic doxorubicin in cellular metabolism. J Pharm Pharmacol, 2016. 68(6): p. 729-41.

12. Octavia, Y., et al., Doxorubicin-induced cardiomyopathy: from molecular mechanisms to therapeutic strategies. J Mol Cell Cardiol, 2012. 52(6): p. 1213-25.

13. Tacar, O. and C.R. Dass, Doxorubicin-induced death in tumour cells and cardiomyocytes: is autophagy the key to improving future clinical outcomes? J Pharm Pharmacol, 2013. 65(11): p. 1577-89.

14. De Angelis, A., et al., Doxorubicin cardiotoxicity and target cells: a broader perspective. Cardio-Oncology, 2016. 2(2).

15. Clerk, A., et al., Regulation of cardiac myocyte cell death. Pharmacol Ther, 2003. 97(3): p. 223-61.

16. Olivetti, G., et al., Apoptosis in the failing human heart. N Engl J Med, 1997. 336(16): p. 113141.

17. Weiner, L.M., Oxygen radicals generation and DNA scission by anticancer and synthetic quinones. Methods Enzymol, 1994. 233: p. 92-105.

18. Korff, S., H.A. Katus, and E. Giannitsis, Differential diagnosis of elevated troponins. Heart, 2006. 92(7): p. 987-93.

19. van Empel, V.P., et al., Myocyte apoptosis in heart failure. Cardiovasc Res, 2005. 67(1): p. 219.

20. Silva, M.T., Secondary necrosis: the natural outcome of the complete apoptotic program. FEBS Lett, 2010. 584(22): p. 4491-9.

21. Leist, M., et al., Intracellular adenosine triphosphate (ATP) concentration: a switch in the decision between apoptosis and necrosis. J Exp Med, 1997. 185(8): p. 1481-6.

22. Ventura-Clapier, R., et al., Bioenergetics of the failing heart. Biochim Biophys Acta, 2011. 1813(7): p. 1360-72.

23. Saraste, A., et al., Cardiomyocyte apoptosis and progression of heart failure to transplantation. Eur J Clin Invest, 1999. 29(5): p. 380-6. 
24. Wencker, D., et al., A mechanistic role for cardiac myocyte apoptosis in heart failure. J Clin Invest, 2003. 111(10): p. 1497-504.

25. Zhang, S., et al., Identification of the molecular basis of doxorubicin-induced cardiotoxicity. Nat Med, 2012. 18(11): p. 1639-42.

26. Green, D.R. and G. Kroemer, The pathophysiology of mitochondrial cell death. Science, 2004. 305(5684): p. 626-9.

27. Bae, S., et al., Delayed activation of caspase-independent apoptosis during heart failure in transgenic mice overexpressing caspase inhibitor CrmA. Am J Physiol Heart Circ Physiol, 2010. 299(5): p. H1374-81.

28. Moreira, A.C., et al., Mitochondrial apoptosis-inducing factor is involved in doxorubicininduced toxicity on H9c2 cardiomyoblasts. Biochim Biophys Acta, 2014. 1842(12 Pt A): p. 2468-78.

29. Lyu, Y.L., et al., Topoisomerase Ilbeta mediated DNA double-strand breaks: implications in doxorubicin cardiotoxicity and prevention by dexrazoxane. Cancer Res, 2007. 67(18): p. 883946.

30. Huber, H.J., et al., Glucose metabolism determines resistance of cancer cells to bioenergetic crisis after cytochrome-c release. Mol Syst Biol, 2011. 7: p. 470.

31. Schmid, J., et al., Systems analysis of cancer cell heterogeneity in caspase-dependent apoptosis subsequent to mitochondrial outer membrane permeabilisation. J Biol Chem, 2012.

32. Sawyer, D.B., Anthracyclines and heart failure. N Engl J Med, 2013. 368(12): p. 1154-6.

33. Xiong, S., et al., Loss of Mdm4 results in p53-dependent dilated cardiomyopathy. Circulation, 2007. 115(23): p. 2925-30.

34. Villunger, A., et al., p53-and drug-induced apoptotic responses mediated by BH3-only proteins puma and noxa. Science, 2003. 302(5647): p. 1036-8.

35. Li, D., et al., Doxorubicin-induced apoptosis in H9c2 cardiomyocytes by NF-kappaB dependent PUMA upregulation. Eur Rev Med Pharmacol Sci, 2013. 17(17): p. 2323-9.

36. Toth, A., et al., Targeted deletion of Puma attenuates cardiomyocyte death and improves cardiac function during ischemia-reperfusion. Am J Physiol Heart Circ Physiol, 2006. 291(1): p. H52-60.

37. Huang, J., et al., $B c l-x L$ gene transfer protects the heart against ischemia/reperfusion injury. Biochem Biophys Res Commun, 2003. 311(1): p. 64-70.

38. Imahashi, K., et al., Transgenic expression of Bcl-2 modulates energy metabolism, prevents cytosolic acidification during ischemia, and reduces ischemia/reperfusion injury. Circ Res, 2004. 95(7): p. 734-41.

39. Hochhauser, E., et al., Bax ablation protects against myocardial ischemia-reperfusion injury in transgenic mice. Am J Physiol Heart Circ Physiol, 2003. 284(6): p. H2351-9.

40. Konorev, E.A., S. Vanamala, and B. Kalyanaraman, Differences in doxorubicin-induced apoptotic signaling in adult and immature cardiomyocytes. Free Radic Biol Med, 2008. 45(12): p. 1723-8.

41. Jeremias, I., K. Stahnke, and K.M. Debatin, CD95/Apo-1/Fas: independent cell death induced by doxorubicin in normal cultured cardiomyocytes. Cancer Immunol Immunother, 2005. 54(7): p. 655-62.

42. Liao, X., et al., Involvement of death receptor signaling in mechanical stretch-induced cardiomyocyte apoptosis. Life Sci, 2005. 77(2): p. 160-74.

43. Hanahan, D. and R.A. Weinberg, Hallmarks of cancer: the next generation. Cell, 2011. 144(5): p. 646-74.

44. Huber, H.J., R.G. McKiernan, and J.H. Prehn, Harnessing system models of cell death signalling for cytotoxic chemotherapy: towards personalised medicine approaches? J Mol Med (Berl), 2014. 92(3): p. 227-37.

45. Ni Chonghaile, T., et al., Pretreatment mitochondrial priming correlates with clinical response to cytotoxic chemotherapy. Science, 2011. 334(6059): p. 1129-33. 
46. Potts, M.B., et al., Reduced Apaf-1 levels in cardiomyocytes engage strict regulation of apoptosis by endogenous XIAP. J Cell Biol, 2005. 171(6): p. 925-30.

47. Potts, P.R., et al., Critical function of endogenous XIAP in regulating caspase activation during sympathetic neuronal apoptosis. J Cell Biol, 2003. 163(4): p. 789-99.

48. Shi, J., et al., Downregulation of doxorubicin-induced myocardial apoptosis accompanies postnatal heart maturation. Am J Physiol Heart Circ Physiol, 2012. 302(8): p. H1603-13.

49. Nitobe, J., et al., Reactive oxygen species regulate FLICE inhibitory protein (FLIP) and susceptibility to Fas-mediated apoptosis in cardiac myocytes. Cardiovasc Res, 2003. 57(1): p. $119-28$.

50. Yamaoka, M., et al., Apoptosis in rat cardiac myocytes induced by Fas ligand: priming for Fasmediated apoptosis with doxorubicin. J Mol Cell Cardiol, 2000. 32(6): p. 881-9.

51. Ibe, W., et al., Cardiomyocyte apoptosis is related to left ventricular dysfunction and remodelling in dilated cardiomyopathy, but is not affected by growth hormone treatment. Eur J Heart Fail, 2007. 9(2): p. 160-7.

52. Badorff, C., et al., Fas receptor signaling inhibits glycogen synthase kinase 3 beta and induces cardiac hypertrophy following pressure overload. J Clin Invest, 2002. 109(3): p. 373-81.

53. Purevjav, E., et al., Myocardial Fas ligand expression increases susceptibility to AZT-induced cardiomyopathy. Cardiovasc Toxicol, 2007. 7(4): p. 255-63.

54. Lindner, A.U., et al., Systems Analysis of BCL2 Protein Family Interactions Establishes a Model to Predict Responses to Chemotherapy. Cancer Res, 2013. 73(2): p. 519-28.

55. Certo, M., et al., Mitochondria primed by death signals determine cellular addiction to antiapoptotic BCL-2 family members. Cancer cell, 2006. 9(5): p. 351-65.

56. Spallarossa, P., et al., Carvedilol prevents doxorubicin-induced free radical release and apoptosis in cardiomyocytes in vitro. J Mol Cell Cardiol, 2004. 37(4): p. 837-46.

57. Childs, A.C., et al., Doxorubicin treatment in vivo causes cytochrome $C$ release and cardiomyocyte apoptosis, as well as increased mitochondrial efficiency, superoxide dismutase activity, and Bcl-2:Bax ratio. Cancer Res, 2002. 62(16): p. 4592-8.

58. Donath, S., et al., Apoptosis repressor with caspase recruitment domain is required for cardioprotection in response to biomechanical and ischemic stress. Circulation, 2006. 113(9): p. 1203-12.

59. Thompson, K.L., et al., Early alterations in heart gene expression profiles associated with doxorubicin cardiotoxicity in rats. Cancer Chemother Pharmacol, 2010. 66(2): p. 303-14.

60. Tait, S.W. and D.R. Green, Mitochondria and cell death: outer membrane permeabilization and beyond. Nat Rev Mol Cell Biol, 2010. 11(9): p. 621-32.

61. Nicholls, D.G. and S.L. Budd, Mitochondria and neuronal survival. Physiol Rev, 2000. 80(1): p. 315-60.

62. Warburg, O., On the origin of cancer cells. Science, 1956. 123(3191): p. 309-14.

63. Neubauer, S., The failing heart--an engine out of fuel. N Engl J Med, 2007. 356(11): p. 114051.

64. van Bilsen, M., et al., Metabolic remodelling of the failing heart: the cardiac burn-out syndrome? Cardiovasc Res, 2004. 61(2): p. 218-26.

65. Shao, D. and R. Tian, Glucose Transporters in Cardiac Metabolism and Hypertrophy. Compr Physiol, 2015. 6(1): p. 331-51.

66. Hrelia, S., et al., Doxorubicin induces early lipid peroxidation associated with changes in glucose transport in cultured cardiomyocytes. Biochim Biophys Acta, 2002. 1567(1-2): p. 1506.

67. Williams, D.W., et al., Local caspase activity directs engulfment of dendrites during pruning. Nat Neurosci, 2006. 9(10): p. 1234-6.

68. Rehm, M., et al., Systems analysis of effector caspase activation and its control by X-linked inhibitor of apoptosis protein. Embo J, 2006. 25(18): p. 4338-49.

69. Sheridan, C. and S.J. Martin, Commitment in apoptosis: slightly dead but mostly alive. Trends Cell Biol, 2008. 18(8): p. 353-7. 
70. Putinski, C., et al., Intrinsic-mediated caspase activation is essential for cardiomyocyte hypertrophy. Proc Natl Acad Sci U S A, 2013. 110(43): p. E4079-87.

71. Narula, J., et al., Apoptosis and the systolic dysfunction in congestive heart failure. Story of apoptosis interruptus and zombie myocytes. Cardiol Clin, 2001. 19(1): p. 113-26.

72. Goormaghtigh, E., et al., Evidence of a specific complex between adriamycin and negativelycharged phospholipids. Biochim Biophys Acta, 1980. 597(1): p. 1-14.

73. Vasquez-Vivar, J., et al., Endothelial nitric oxide synthase-dependent superoxide generation from adriamycin. Biochemistry, 1997. 36(38): p. 11293-7.

74. Xu, M.F., et al., Effects by doxorubicin on the myocardium are mediated by oxygen free radicals. Life Sci, 2001. 68(8): p. 889-901.

75. Hashem, S.I., et al., Brief Report: Oxidative Stress Mediates Cardiomyocyte Apoptosis in a Human Model of Danon Disease and Heart Failure. Stem Cells, 2015. 33(7): p. 2343-50.

76. Sinha, K., et al., Oxidative stress: the mitochondria-dependent and mitochondria-independent pathways of apoptosis. Arch Toxicol, 2013. 87(7): p. 1157-80.

77. Vedam, K., et al., Role of heat shock factor-1 activation in the doxorubicin-induced heart failure in mice. Am J Physiol Heart Circ Physiol, 2010. 298(6): p. H1832-41.

78. Tacar, O., et al., Cardiomyocyte apoptosis vs autophagy with prolonged doxorubicin treatment: comparison with osteosarcoma cells. J Pharm Pharmacol, 2015. 67(2): p. 231-43.

79. Dhingra, R., et al., Ellagic Acid Suppresses Mitophagy- Induced Necrotic Cell Death During Doxorubicin Cardiotoxicity. 2016.

80. Weiner, A., et al., The Role of Mitophagy in Doxorubicin-Induced Cardiomyocyte Death, E.B. Meeting, Editor. 2016, Faseb J.

81. Wang, S., et al., Doxorubicin induces apoptosis in normal and tumor cells via distinctly different mechanisms. intermediacy of $\mathrm{H}(2) \mathrm{O}(2)$ - and p53-dependent pathways. J Biol Chem, 2004. 279(24): p. 25535-43.

82. Ichim, G. and S.W. Tait, A fate worse than death: apoptosis as an oncogenic process. Nat Rev Cancer, 2016. 16(8): p. 539-48.

83. Branco, A.F., et al., Differentiation-dependent doxorubicin toxicity on H9c2 cardiomyoblasts. Cardiovasc Toxicol, 2012. 12(4): p. 326-40.

84. Hector, S., et al., Clinical application of a systems model of apoptosis execution for the prediction of colorectal cancer therapy responses and personalisation of therapy. Gut (in press), 2012.

85. Huber, H.J., et al., Mathematical modelling of the mitochondrial apoptosis pathway BBA Mol. Cell Res., 2011. $x(x)$.

86. Junjing, Z., Z. Yan, and Z. Baolu, Scavenging effects of dexrazoxane on free radicals. J Clin Biochem Nutr, 2010. 47(3): p. 238-45.

87. Dirkx, E., P.A. da Costa Martins, and L.J. De Windt, Regulation of fetal gene expression in heart failure. Biochim Biophys Acta, 2013. 1832(12): p. 2414-24.

88. Vandenwijngaert, S., et al., Increased cardiac myocyte PDE5 levels in human and murine pressure overload hypertrophy contribute to adverse LV remodeling. PLoS One, 2013. 8(3): p. e58841.

89. Christofk, H.R., et al., The M2 splice isoform of pyruvate kinase is important for cancer metabolism and tumour growth. Nature, 2008. 452(7184): p. 230-3.

90. Vander Heiden, M., L. Cantley, and C. Thomson, Understanding the Warburg Effect: The Metabolic Requirements of Cell Proliferation. Science, 2009. 324(1029): p. 1029-1033. 